\title{
Pathological Fracture: An Unusual Presentation in Childhood Chronic Myeloid Leukemia
}

\author{
Mururul Aisyi ${ }^{1}$, Ayu Hutami Syarif ${ }^{2}$, Anita Meisita ${ }^{1}$, Agus Kosasih $^{3}$, Achmad Basuki ${ }^{4}$, Resti \\ Mulya Sari ${ }^{5}$ \\ ${ }^{1}$ Department of Pediatric Hematology Oncology, Indonesia National Cancer Center Dharmais Cancer Hospital \\ ${ }^{2}$ Research and Development Department, Indonesia National Cancer Center Dharmais Cancer Hospital \\ ${ }^{3}$ Department of Clinical Pathology, Indonesia National Cancer Center Dharmais Cancer Hospital \\ ${ }^{4}$ Department of Orthopedi, Indonesia National Cancer Center Dharmais Cancer Hospital \\ ${ }^{5}$ Department of Hematology \& Medical Oncology, Indonesia National Cancer Center Dharmais Cancer Center
}

\section{ARTICLE INFO}

Received : 06 September 2019

Reviewed : 08 October 2019

Accepted : 12 December 2019

Keywords:

pediatric, fracture, nilotinib, leukemia

\author{
*Corresponding author: \\ Mururul Aisyi \\ Department of Pediatric Hematology \\ Oncology, Indonesia National Cancer \\ Center Dharmais Cancer Hospital, \\ Jakarta, Indonesia. \\ aci97nabil@yahoo.com
}

\begin{abstract}
A BSTRACT
Introduction: Chronic Myeloid Leukemia is a hematological malignancy driving from myeloproliferative process. It is typified by the presence of the Philadelphia chromosome manifesting in certain distinct complications, including pathological fracture. Pathological fracture is recognized as an extramedullary disease that occurs as a result of transformation of $\mathrm{CML}$ into blast crisis phase.
\end{abstract}

Case Presentation: Here, we report a case of pediatric male CML. After being failed with imatinib therapy, he turned to nilotinib and was unable to achieve a major molecular response. He presented with high blast count and pain in the left arm. He was diagnosed with pathological fracture and blast crisis phase CML. Taken the young age and displacement of fracture into consideration, he was conservatively treated by a combination of immobilization and a higher dose of targeted therapy, nilotinib. The 2-month evaluation revealed clinical union and reduction of blast cells.

Conclusions: Regarding the minimal displacement and age presentation, pathological fracture in pediatric CML requires non-invasive treatment and optimization of antileukemic therapy.

\section{INTRODUCTION}

Chronic Myeloid Leukemia (CML) is a myeloproliferative neoplasm marked by the presence of Philadelphia (Ph) chromosome, a translocation of chromosomes 22 and 9. CML may present at any age ranging from adolescence to adulthood. It is divided into three different phases: chronic, accelerated, and blast crisis. Most cases are commonly found at chronic phase [1,2]. By using the Ph chromosome (BCR-ABL), CML can be diagnosed precisely and be targeted to the specific inhibitor, Tyrosine Kinase Inhibitor (TKI), in addition to the monitoring and response evaluation after TKI treatment.[3].

Bone involvement in $\mathrm{CML}$ rarely occurs. The radiographical findings vary from osteoporosis, osteolytic, osteoblastic lesions, chloroma, bone infarct, to arthritis $[4,5]$. It was reported only in $3 \%$ to $5 \%$ of $\mathrm{CML}$ cases and related to the impending of blast crisis phase [6]. Hypothetically, the fracture is manifested from metastatic lesions or co-existing solid tumors [7]. Another study correlated it with medication history such as steroid
(4) or TKI [8-10]. Pathological fracture in CML is even rarely observed. During the last few decades, only two cases had been reported. Our case report highlights an atypical presentation and management of fracture in CML in our institution.

\section{CASE PRESENTATION}

An 18-year old male admitted to the hospital complaining of pain at the left shoulder in the last 4 days. He was also unable to move his left shoulder. There was no history of trauma or fall. He has been diagnosed with CML since 2013 and had taken imatinib for 4 years. During the follow-up period, the patient returned with hyperleukocytosis, blast count of $27 \%$, and the BCR-ABL ratio of $69.37 \%$ IS. Having those results, the patient was suspected to be resistant to imatinib. Consequently, his medication was changed to nilotinib, starting at a dose of $200 \mathrm{mg}$ once daily. Following the 
two-week administration, blast crisis was improved (7\%) as well as 5-month evaluation of BCR-ABL ratio (1.39\%). However, after one year of nilotinib treatment, the patient failed to achieve major molecular responses with BCR-ABL ratio of $22.7 \%$.

On his admission, he was alert and vital signs were stable. On the physical examination, there was a soft tissue swelling around the left shoulder. A limited range of motion was elicited in his left shoulder due to pain. Complete blood count measurement showed hyperleukocytosis (white blood count 91820/ $\mu \mathrm{L}$ ). The plain x-ray of the left shoulder revealed metastatic lesion and pathological fracture of left neck humerus. Bone scanning showed metastatic lesions in certain areas of the body (Figure 2). The patient was immobilized with an arm sling. Moreover, bone marrow biopsy showed $34 \%$ of blast cells (Figure 3 ) indicating a diagnosis of $\mathrm{CML}$ on the blast crisis phase. Consequently, the patient was given another round of nilotinib with an escalating dose (200 mg twice a day). Since the empirical treatment of the blast crisis in our institution consisting of targeted therapy and the toxic effects of chemotherapy is somewhat of concern, additional chemotherapy was not given to the patient.

After 2 months of receiving those treatments, the patient showed clinical improvement. Routine blood workup showed a normal leukocyte count $(4590 / \mu \mathrm{L})$ and no identifying peripheral blast count. Moreover, his range of motion was improved along with plain radiography depicting site of callus progressivity and reduction in osteolytic lesion. These two combinations indicated a clinical union.
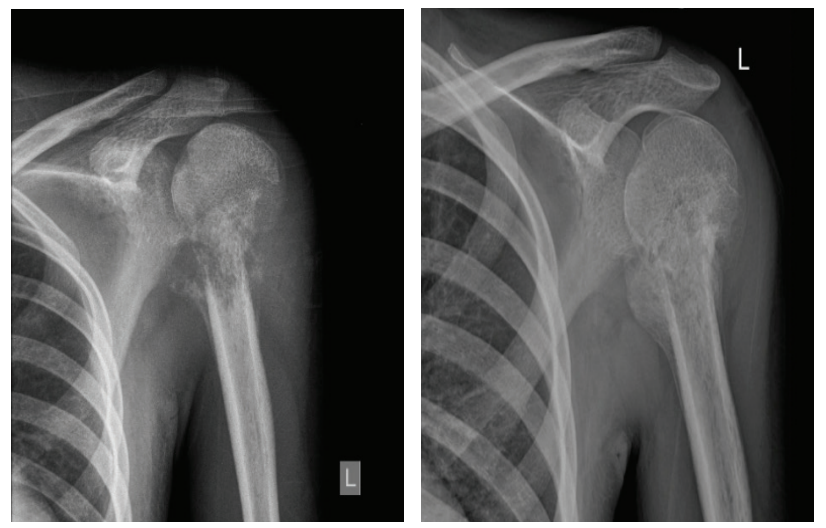

Figure 1. Xray of left shoulder indicating lytic lesion with a pathological fracture in the neck of left humerus (A). 2-month evaluation showed callus formation, indicated by a white arrow sign (B) and improvement in soft tissue swelling. There is no osteolytic and osteoblastic lesion.

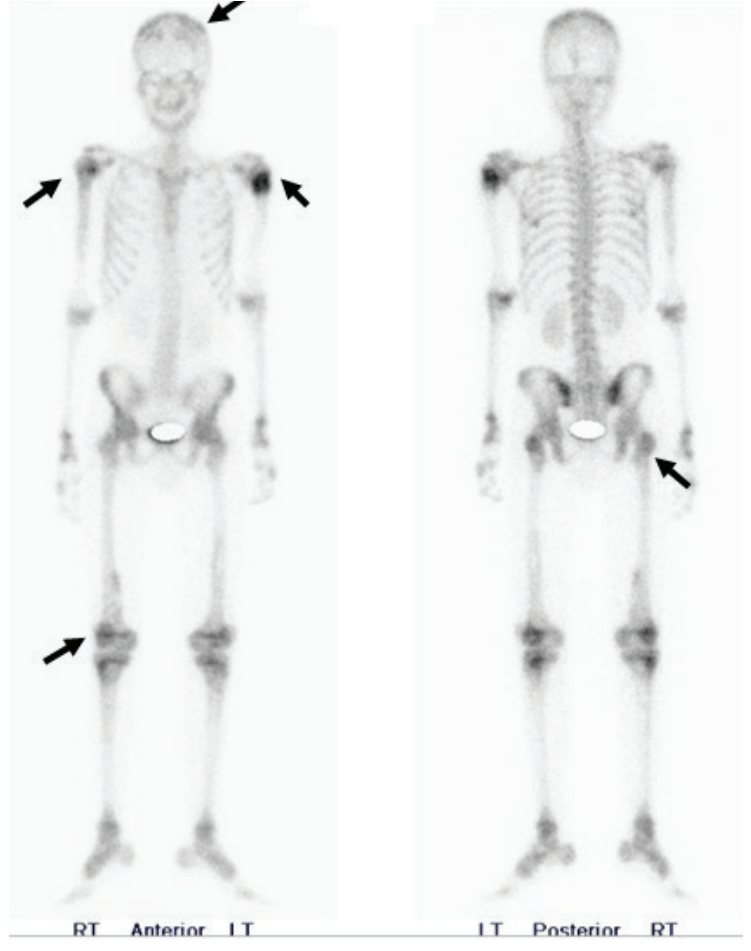

Figure 2. Bone scan showing pathological activity in several bones. (A) from the anterior aspect, this activity indicating several metastatic lesions (black arrow) in the left and right humerus, right distal femur, and bilateral frontoparietal bone. (B) from the posterior aspect, metastatic lesion shown in the left proximal femur
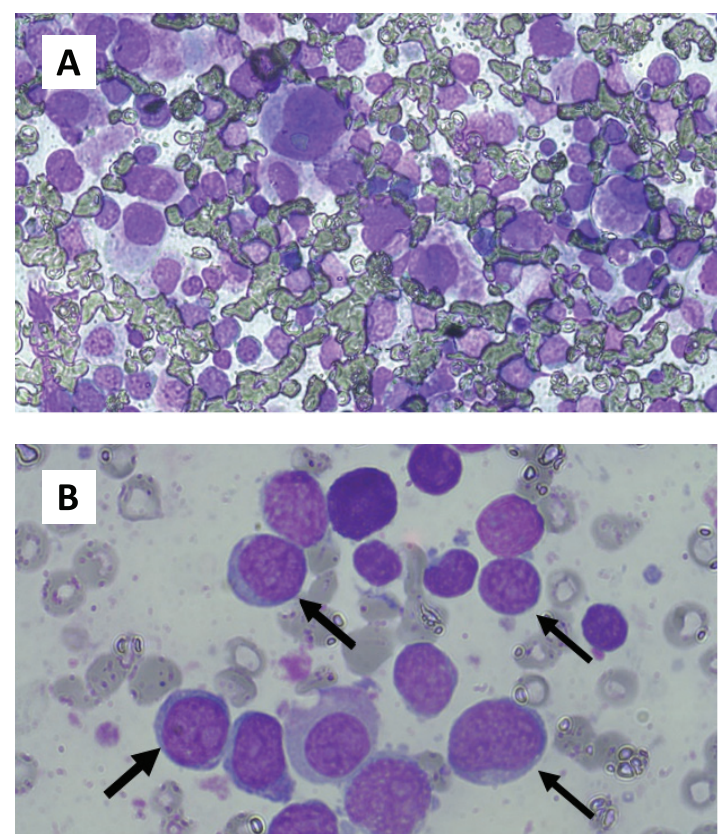

Figure 3. Cytology examination. (A) Low power magnification (400x) of bone marrow staining depicting a hypercellularity with granulopoietic hyperactive basophilia, an increased thrombopoietic activity, and megakaryocytes, and decrease in erythropoiesis. (B) High power magnification (1000x) of smear showing prominent myeloblasts (black arrows) 


\section{DISCUSSION}

The main treatment for CML with positive Philadelphia chromosome includes tyrosine kinase inhibitor as targeted therapy. In this case, imatinib and nilotinib were used accordingly as the patient developed resistance to the former. Nilotinib as a second-line therapy is aimed for the long term or even throughout life medication. The therapeutic response is based on hematologic, cytogenetic, and major molecular responses which are performed in 3,6, 12, 18, and 24 months after starting the therapy. Nevertheless, those serial evaluations were not properly undertaken in this current case due to limited resources. Furthermore, the 12-month evaluation revealed a non-remission disease as the patient returned to the blast crisis phase with fracture of the extremity.

Pathological fracture is a rare manifestation of CML. This patient was the first reported CML case complicated with a pathological fracture in our institution. Of note, only two reports had provided the same case. Yu et al. reported a case of a three-year-old girl experiencing femoral fracture as a manifestation of CML (11]. Another case was from a middle-aged woman who had pathological fracture as a result of myeloblastoma [12]. In our patient, giving the history of $\mathrm{CML}$ and bone scanning results, it can be suggested that the fracture was secondary to malignancy.

Pathological fracture can be caused by either extramedullary infiltration of blast cells which is also known as an extramedullary disease (EMD) or drugrelated complication. The prevalence of EMD was approximately $7-17 \%$, which was mostly occurred in the blast crisis phase (extramedullary blast crisis) [13]. Preferred bone sites were commonly in haematopoietically active areas, such as pelvis, vertebrae, neck of femur, head of humerus, and the skull $[14,15]$. Previously, EMD was reported in the proximal femur and presented with lytic lesion [16]. It was also stated in some reports that the extramedullary blast crisis was considered as an early sign of the blast crisis phase [17-19].

Certain medical treatment was highly associated with the occurrence of bone lesion in CML. In general, glucocorticoid use is a risk factor for osteoporotic fracture [19]. However, the fracture in our patient was described as osteolytic lesion rather than porotic and localized lesion. Besides, other plain x-rays also showed normal density. Consequently, it can be ruled out as the cause of fracture in our patient. Imatinib has been widely studied for its mechanism of altering bone remodeling. Patients receiving imatinib or nilotinib had a decrease in total serum phosphate as well as calcium level. A vitro study demonstrated the inhibitory effect of imatinib to osteoclastogenesis [4]. It also enhanced osteogenic gene expression in favor of osteoblast differentiation and bone formation [21,22]. In contrast, a more recent vivo study in rodents conducted by the same investigator has reported a decrease in bone mass during imatinib therapy [10].

The treatment of pathological fracture aims for stabilization of the fracture and treatment of underlying conditions [23]. The stabilization can be either surgical or non-surgical approach. The nature of the age and fracture characteristic determines the treatment choice in this patient. Considering the remaining open epiphysial plate and minimal displacement, the patient underwent immobilization with arm sling and receiving nilotinib with higher dose instead. Two months of nilotinib treatment and immobilization allowed callus formation. The clinical union was ultimately achieved as peripheral blast cell count considerably low ( $\pm 10-14 \%)$. A similar outcome has also ever been reported by Chua et al. who observed fracture healing along with the bone marrow remission post-chemotherapy in a pediatric aleukemic leukemia case [24].

Although the pathological fracture in our case can be provoked by certain medications such as steroids and TKIs, the mechanism itself was still unclear and debatable. The high number of blast cells after receiving lower dose of nilotinib made our patients tend to become resistant to nilotinib. Furthermore, skeletal thorough radiography is proposed to be a routine workup for CML patients.

\section{CONCLUSIONS}

Regarding the minimal displacement and age presentation in terms of pathological fracture in $\mathrm{CML}$, the treatment relies on the characteristic of fracture and antileukemic therapy. In our case, the minimal displacement and pediatric age encourage the use of immobilization as a fracture-specific treatment.

\section{DECLARATIONS}

\section{Competing interest}

The authors declare no competing interest in this case report.

\section{Acknowledgement}

Nothing to declare. 


\section{REFERENCES}

1. Radich JP, Deininger $\mathrm{M}$, Abboud CN, Altman JK, Berman E, Bhatia R, et al. Chronic myeloid leukemia. JNCCN J Natl Compr Cancer Netw. 2018;16(9):1108-35.

2. Granatowicz A, Piatek $\mathrm{Cl}$, Moschiano E, El-Hemaidi I, Armitage JD, Akhtari M. An overview and update of chronic myeloid leukemia for primary care physicians. Korean J Fam Med. 2015;36(5):197-202.

3. Soverini, S., Bassan, R. \& Lion, T. Treatment and monitoring of Philadelphia chromosome-positive leukemia patients: recent advances and remaining challenges. J Hematol Oncol. 2019;12(39):1-14

4. Sharma SK, Seth T, Mishra P, Mahapatra M. Bone Infarcts in a Case of Chronic Myeloid Leukemia: Chronic Phase. Indian J Hematol Blood Transfus. 2012;28(3):187-8.

5. Solav S, Bhandari R, Solav P. Skeletal scintigraphy manifestations of hematologic disorders. Indian J Nucl Med. 2013;27(1):59.

6. Schabel SI, Tyminski L, Holland RD, Rittenberg GM. The skeletal manifestations of chronic myelogenous leukemia. Skeletal Radiol. 1980;5(3):145-9.

7. Prabhash K, Loknath D, Babu KG, Hasib AG, Biswas S, Bapsy PP. Chronic myeloid leukemia with osteolytic bone involvement [7]. J Assoc Physicians India. 2003;51(JUL):740.

8. Berman E, Nicolaides M, Maki RG, Fleisher M, Chanel $S$, Scheu K, et al. Altered Bone and Mineral Metabolism in Patients Receiving Imatinib Mesylate. N Engl J Med. 2006;354(19):2006-13.

9. Terpos E, Apperley J, Milojkovic D. Imatinib and chronic myeloid leukemia: Close to the bone. Leuk Lymphoma. 2013;54(8):1581-2.

10. O'Sullivan S, Naot D, Callon K, Porteous F, Horne A, Wattie $D$, et al. Imatinib promotes osteoblast differentiation by inhibiting PDGFR signaling and inhibits osteoclastogenesis by both direct and stromal cell-dependent mechanisms. J Bone Miner Res. 2007;22(11):1679-89.

11. Yu H-H, Lu M-Y, Lin D-T, Lin K-H, Tang J-L, Jou S-T. Pathological fracture as a manifestation of extramedullary blastic crisis in chronic myelogenous leukemia: report of one case. Acta Paediatr Taiwan. 2019;47(3):150-4.

12. Ishiyama $T$, Sugimoto $M$, Wakabayashi $Y$, Shiokawa Y, Takaku F. [A case of chronic myelogenous leukemia, whose blastic crisis occurred at the same time of the pathologic fracture of the right femur due to myeloblastoma (author's transl)]. Rinsho Ketsueki. 1981;22(5):661-7.
13. Tsukamoto S, Ota S, Ohwada C, Takeda Y, Takeuchi $M$, Sakaida $E$, et al. Extramedullary blast crisis of chronic myelogenous leukemia as an initial presentation. Leuk Res Reports. 2013;2(2):67-9.

14. Clements DG, Kalmon EH. Chronic Myelogenous Leukemia: Unusual Bone Changes in an Adult. Radiology. 2014;67(3):399-403.

15. Ginsberg V, Friedmann A, Meyer L. Infiltration of Bone with Spontaneous Fracture. Jama Network. 2015. p. 4-7.

16. Maloisel F, Favre G, Mahmal L, Zamfir A, Andrès E. Isolated lytic bone lesion as extramedullary disease in chronic myelogenous leukemia: A report of three new cases. Eur J Intern Med. 2005;16(4):288-90.

17. Kantarjian HM, Keating MJ, Talpaz M, Walters RS, Smith TL, Cork A, et al. Chronic myelogenous leukemia in blast crisis. Analysis of 242 patients. Am J Med. 1987;83(3):445-54.

18. Terjanian T, Kantarjian H, Keating M, Talpaz M, McCredie K, Freireich EJ. Clinical and prognostic features of patients with Philadelphia chromosomepositive chronic myelogenous leukemia and extramedullary disease. Cancer. 1987;59(2):297-300.

19. Inverardi D, Lazzarino M, Morra E, Bernasconi $P$, Merante S, Canevari A, et al. Extramedullary disease in $\mathrm{Ph}^{\prime}$-positive chronic myelogenous leukemia: frequency, clinical features and prognostic significance. Haematologica. 1990;75(2):146-8.

20. Farmer S, Horváth-Puhó $E$, Vestergaard $H$, Hermann $A P$, Frederiksen $H$. Chronic myeloproliferative neoplasms and risk of osteoporotic fractures; a nationwide population-based cohort study. $\mathrm{Br} \mathrm{J}$ Haematol. 2013;163(5):603-10.

21. Fitter S, Dewar AL, Kostakis $P$, To LB, Hughes TP, Roberts $\mathrm{MM}$, et al. Long-term imatinib therapy promotes bone formation in CML patients. Blood. 2008;111(5):2538-47.

22. O'Sullivan S, Naot D, Callon KE, Watson M, Gamble $G D$, Ladefoged $M$, et al. Imatinib mesylate does not increase bone volume in vivo. Calcif Tissue Int. 2011;88(1):16-22.

23. Canavese F, Samba A, Rousset M. Pathological fractures in children: Diagnosis and treatment options. Orthop Traumatol Surg Res. 2016;102(1):S149-59.

24. Chua GT, Chow KC, So JCC, Cheuk DKL. Aleukaemic leukaemia presenting with pathological fracture. BMJ Case Rep. 2014;2-5. 